\title{
Direct visual servoing framework based on optimal control for redundant joint structures
}

\author{
Jorge Pomares ${ }^{1, \#}$, Carlos A. Jara ${ }^{1}$, Javier Pérez'and Fernando Torres ${ }^{1}$ \\ 1 Department of Physics, Systems Engineering and Signal Processing, University of Alicante, Carretera de San Vicente del Raspeig, Alicante, Spain, 03690 (Zip code) \\ \# Corresponding Author / E-mail: jpomares@ua.es, TEL: +34-965903400, FAX: +34-965909750 \\ KEYWORDS : Visual servoing, Control framework, Chaos control, Robotics
}

\begin{abstract}
This paper presents a new framework based on optimal control to define new dynamic visual controllers to carry out the guidance of any serial link structure. The proposed general method employs optimal control to obtain the desired behaviour in the joint space based on an indicated cost function which determines how the control effort is distributed over the joints. The proposed approach allows the development of new direct visual controllers for any mechanical joint system with redundancy. Finally, authors show experimental results and verifications on a real robotic system for some derived controllers obtained from the control framework.
\end{abstract}

Manuscript received: August XX, 201X / Accepted: August XX, 201X

\section{Introduction}

Recently, new industrial robot manipulators include kinematic redundant structures in order to face complex and/or multiple tasks in unstructured and/or dynamic environments. This redundancy provides robotic systems with a large number of degrees of freedom (dof) performing complex joint structures. These mechanical structures both overcome intrinsic limitations of the mechanical construction and provide more flexibility during the tasks' performance. In the field of manufacturing processes, requirements such as the complexity of geometric shapes, the accuracy needed in the finished piece and the high speed required for the machining processes have focused the use of redundant joint structures ${ }^{1,2}$. However, controlling a redundant system during this type of tasks involves not only the correct end-effector motion, but also the achievement of correct jointstabilization movements. This paper presents a new optimal control framework for redundant joint structures where the task to be performed is specified in the image space. The classical image-based controllers determine the required camera velocities in order to position the robot with respect a reference object ${ }^{3}$. Using this last approach, the internal robot controller computes the joint torques in order to achieve the previously mentioned camera velocities. However, the framework proposed in this paper to perform the control of the joint structure guidance is based on direct visual servoing. By means of direct visual servo, the internal control loop of servo motors is removed and the visual servo control is employed to stabilize the robot ${ }^{4}$. The result is a faster and more accurate control (C) KSPE and Springer 2011 that reacts more quickly to abrupt changes in the image trajectories, a very suitable feature for non-contact machining processes. Moreover, the approach presented is based on an optimal control framework and it is employed to control redundant joint structures taking into account the robot dynamics. This way, this control approach considers the optimization of the motor signals or torques sent to the mechanical system during visual control tasks. Using this framework, several new direct visual controllers can be obtained for any mechanical system with redundancy, which offers a useful unification methodology. A precision study of different controllers derived from the framework is presented using a tool based on a joint structure whose main use will be to perform complex shapes in some noncontact machining processes such as laser or water cutting.

The control of redundant mechanical systems has been a topic research in the last years, where many authors have proposed different control algorithms based on dynamics. It is worth pointing out the work presented by Kathib ${ }^{5}$, which proposed a dynamic control scheme for redundant robots using the pseudo-inverse of the Jacobian matrix. Other works such as these presented by $\mathrm{Xian}^{6}$ and Zergeroglu ${ }^{7}$, are based on a nonlinear controller which ensured an asymptotic tracking in the Cartesian space. Tatlicioblu ${ }^{8}$ and $\operatorname{Lin}^{9}$ presented the application of another kind of controllers such adaptive and optimal control in the path planning. Other recent approaches are focused in neural network technology because of their learning capabilities and parallel computing. In this field, most of the contributions are in charge of compensating both the nonlinearities ${ }^{10}$ and structured/unstructured uncertainties of the system ${ }^{11}$. The above 
commented approaches are based on position, orientation and/or velocity control in the path tracking. However, as it is previously indicated, the proposed framework employs image information for the robot guidance. Therefore, the use of this framework allows us to derive new visual controllers to track complex shapes in the image space using any redundant joint structure, guaranteeing that the correct joint motion is obtained.

In applications such as laser-cutting, the redundant machine must often track repetitive or quasi-repetitive trajectories. This can produce a non-periodic joint motion or chaotic behavior. As it is shown in the results section, a chaotic behavior in the joint space can be obtained while using a classical visual servoing system. In this case, the robot-end tracks the image trajectory correctly but an unpredictable and non-periodic motion is generated in the joint space. This non-repeatability in the joint space limits the practical applications of the visual controller. In previous works ${ }^{12}$, the authors have integrated chaos compensation in classical dynamic visual controllers in order to obtain a periodic joint trajectory when the robot-end also tracks a periodic image trajectory. This last aspect guarantees a smoother joint behavior and increases the safety by obtaining predictable trajectories. In this paper, the chaos controller is also integrated in the proposed framework. Therefore, the new controllers derived from the framework also include chaos compensation. In the section where the experimental results are presented, a precision study is included in order to evaluate the new direct visual controllers.

The paper is organized as follows. Section 2 explains the optimal control framework employed in the image tracking of the redundant joint structure. Section 3 shows the required modifications for redundancy resolution and chaos control, and the general dynamic visual servoing framework is defined. Section 4, some new imagebased controllers are obtained from the dynamic visual servoing framework and the experimental results are presented. The final section reports some important conclusions.

\section{Optimal visual control for redundant joint structures}

This section shows the proposed control framework to guide redundant joint structures using visual information. In order to accurately explain the control framework, this section is divided in five subsections. Firstly, the kinematics and dynamics formulation of a joint structure is defined. Secondly, the visual servoing concepts employed in the framework, are described. Afterwards, the optimal control approach applied for visual servoing is explained. Next, the image trajectory is described as a task constrain. Finally, the required modifications of the control framework for redundancy resolution and chaos control are presented.

\subsection{Kinematics and dynamics of the joint structure}

The differential kinematics of a joint structure with $\mathrm{n}$ dof establishes the relationship between the joint velocities and the corresponding end-effector velocity. This relationship can be given as follows:

$$
\dot{\mathbf{r}}=\mathbf{J}_{\mathrm{r}}(\mathbf{q}) \dot{\mathbf{q}}
$$

where $\mathbf{r} \in \mathfrak{R} \eta$ is the pose of the camera located at the robot endeffector, being $\eta$ the dimension of the space where the path tracking is performed, $\dot{\mathbf{r}}$ is the Cartesian end-effector velocity, $\mathbf{q} \in \mathfrak{R}^{\mathrm{nx} 1}$ is a vector of generalized joint coordinates, $\dot{\mathbf{q}} \in \mathfrak{R}^{\mathrm{n} \times 1}$ represents the joint velocities, and $\mathbf{J}_{\mathbf{r}}(\mathbf{q})$ is the manipulator Jacobian matrix.

In regard to the dynamics, the well-known model of a serial nlink rigid mechanism, in absence of friction, can be written as:

$$
\boldsymbol{\tau}=\mathbf{M}(\mathbf{q}) \ddot{\mathbf{q}}+\mathbf{C}(\mathbf{q}, \dot{\mathbf{q}})+\mathbf{G}(\mathbf{q})
$$

where $\ddot{\mathbf{q}} \in \mathfrak{R}^{\mathrm{nx} 1}$ is the joint accelerations, $\quad \mathbf{M}(\mathbf{q}) \in \mathfrak{R}^{\mathrm{nxn}}$ is the symmetric positive definite manipulator inertia matrix, $\mathbf{C}(\mathbf{q}, \dot{\mathbf{q}}) \in \mathfrak{R}^{\mathrm{nx} 1}$ denotes the vector of centripetal and Coriolis forces, and $\mathbf{G}(\mathbf{q}) \in \mathfrak{R}^{\mathrm{nx} 1}$ is the gravitational force. Finally, $\boldsymbol{\tau} \in \mathfrak{R}^{\mathrm{nx} 1}$ is the vector of applied motor commands (i.e. torques or forces). In order to simplify this equation, we can write the dynamics as follows:

$$
\mathbf{M}(\mathbf{q}) \ddot{\mathbf{q}}=\boldsymbol{\tau}+\mathbf{F}_{\mathrm{cg}}(\mathbf{q}, \dot{\mathbf{q}})
$$

where $\mathbf{F}_{\mathrm{cg}}(\mathbf{q}, \dot{\mathbf{q}})=-\mathbf{C}(\mathbf{q}, \dot{\mathbf{q}})-\mathbf{G}(\mathbf{q})$

\subsection{Visual servo control}

Visual servo control techniques ${ }^{3}$ allow the guidance of a joint robot using visual information, typically using an eye-in-hand configuration where a camera is held by the mechanism end-effector. The relationship between velocities in camera image space, $\dot{\mathbf{s}}_{\mathrm{r}}$, and end-effector velocity, $\dot{\mathbf{r}}$, is represented by:

$$
\dot{\mathbf{s}}_{\mathrm{r}}=\mathbf{L}_{\mathrm{s}}(\mathbf{s}) \dot{\mathbf{r}}
$$

where $\mathbf{L}_{\mathbf{s}}(\mathbf{s})$ is the interaction matrix ${ }^{3}$. From this relation and Equation (1), the image space velocity $\dot{\mathbf{s}}_{\mathrm{r}}$ can be related to joint space velocity $\dot{\mathbf{q}}$ by means of the following relationship:

$$
\dot{\mathbf{s}}_{\mathrm{r}}=\mathbf{L}_{\mathrm{s}}(\mathbf{s}) \cdot \mathbf{J}_{\mathrm{r}}(\mathbf{q}) \dot{\mathbf{q}}=\mathbf{L}_{\mathrm{J}}(\mathbf{q}, \mathbf{s}) \dot{\mathbf{q}}
$$

where $\mathbf{L}_{\mathbf{J}}=\mathbf{L}_{\mathbf{J}}(\mathbf{q}, \mathbf{s})$ is the Jacobian matrix mapping from joint space to image space. This matrix relates differential changes in joint configuration of the robot to differential changes in the observed image feature parameters.

The image acceleration or second time derivative of $\mathbf{S}$ is obtained by differentiating Equation (5) with respect to the time:

$$
\ddot{\mathbf{s}}_{\mathrm{r}}=\mathbf{L}_{\mathrm{J}} \ddot{\mathbf{q}}+\dot{\mathbf{L}}_{\mathrm{J}} \dot{\mathbf{q}}
$$

The variable $\ddot{\mathbf{s}}_{\mathrm{r}}$ denotes the reference image accelerations of our controller based on image space.

\subsection{Optimal control approach}

The dynamic model of a serial-link robot represented by Equation (2) has been used in different approaches to control a robotic system for tracking ${ }^{12}$. Following this idea, the approach proposed by Udwadia $^{14}$ gave a new perspective about tracking based on optimal control for nonlinear mechanical systems. This approach will be used in this paper in order to perform the visual tracking of a redundant joint structure.

Basically, the control approach suggested by Udwadia ${ }^{14}$ supposes a system with $\mathrm{m}$ constrains (holonomic and/or non-holonomic) which 
represent the task for the robot to be described. The time derivate of these constraints is represented by the following equation:

$$
\mathbf{A}(\mathbf{q}, \dot{\mathbf{q}}, \mathrm{t}) \ddot{\mathbf{q}}=\mathbf{b}(\mathbf{q}, \dot{\mathbf{q}}, \mathrm{t})
$$

where $\mathbf{A}(\mathbf{q}, \dot{\mathbf{q}}, \mathrm{t}) \in \mathfrak{R}^{\mathrm{mxn}}$ and $\mathbf{b}(\mathbf{q}, \dot{\mathbf{q}}, \mathrm{t}) \in \mathfrak{R}^{\mathrm{mx} 1}$ are the matrix and the vector obtained, respectively. The optimal controller treats to minimize the control torques of the mechanical system while performing a specific task taking into account the following function cost:

$$
\mathbf{\Omega}(\mathrm{t})=\boldsymbol{\tau}^{\mathrm{T}} \mathbf{W}(\mathrm{t}) \boldsymbol{\tau}
$$

where $\mathbf{W}(\mathrm{t})$ is a time-dependent weight matrix. The function control that minimizes $\boldsymbol{\Omega}(\mathrm{t})$ of the mechanical system based on the dynamics model expressed in Equation (3) while performing the task described in Equation (7) is given by (for the sake of clarity the time and joint dependences are not indicated):

$$
\boldsymbol{\tau}=\mathbf{W}^{-1 / 2}\left(\mathbf{A} \mathbf{M}^{-1} \mathbf{W}^{-1 / 2}\right)^{+} \cdot\left(\mathbf{b}-\mathbf{A} \mathbf{M}^{-1} \mathbf{F}_{\mathrm{cg}}\right)
$$

where $\mathbf{M}$ is the inertia matrix and the symbol + denotes the pseudoinverse for a general matrix. As it can be seen in Equation (9), the matrix $\mathbf{W}$ is an important depending variable in the control law and determines how the control effort is distributed over the joints.

Equation (6), which describes the image task of the redundant robot, can be expressed into the form of Equation (7):

$$
\mathbf{L}_{\mathrm{J}} \ddot{\mathbf{q}}=\ddot{\mathbf{s}}_{\mathrm{r}}-\dot{\mathbf{L}}_{\mathrm{J}} \dot{\mathbf{q}}
$$

This way, the task constrains are defined by the following relationships:

$$
\begin{aligned}
& \mathbf{A}=\mathbf{L}_{\mathrm{J}} \\
& \mathbf{b}=\ddot{\mathbf{s}}_{\mathrm{r}}-\dot{\mathbf{L}}_{\mathrm{J}} \dot{\mathbf{q}}
\end{aligned}
$$

Therefore, with this definition of $\mathbf{A}$ and $\mathbf{b}$, the optimal control will minimize the torques of the joint structure while performing a tracking in the image space. The final control law can be obtained replacing these variables into the function that minimizes the motor signals described by Equation (9):

$$
\boldsymbol{\tau}=\mathbf{W}^{-1 / 2}\left(\mathbf{L}_{\mathrm{J}} \mathbf{M}^{-1} \mathbf{W}^{-1 / 2}\right)^{+} \cdot\left(\ddot{\mathbf{s}}_{\mathrm{r}}-\dot{\mathbf{L}}_{\mathrm{J}} \dot{\mathbf{q}}-\mathbf{L}_{\mathrm{J}} \mathbf{M}^{-1} \mathbf{F}_{\mathrm{cg}}\right)
$$

As it can be seen, the visual controller represented by (12) depends implicitly on the weighting matrix $\mathbf{W}$ and different value $\mathrm{s}$ of this matrix can simplify the product $\left(\mathbf{L}_{\mathbf{J}} \mathbf{M}^{-1} \mathbf{W}^{-1 / 2}\right)^{+}$and consequently, the control law. Additionally, different visual servoing control laws can be obtained with different values of $\mathbf{W}$, performing a new optimal control framework for the visual tracking of redundant joint structures.

\subsection{Task description using an image trajectory}

The optimal control approach described in the previous subsection will be employed to track an image trajectory taking into account the robot dynamics. As shown, the tracked trajectory is defined and expressed as a set of constraints following Equation (7) (see Equation (11)). In this subsection, the definition of our reference control $\ddot{\mathbf{s}}_{\mathrm{r}}$ is described considering an eye-in-hand camera system which extracts a set of $\mathrm{k}$ image feature points.
Let $\mathbf{s}=\left[f_{1 \mathrm{x}}, f_{1 \mathrm{y}}, f_{2 \mathrm{x}}, f_{2 \mathrm{y}}, \ldots, f_{\mathrm{kx}}, f_{\mathrm{ky}}\right]^{\mathrm{T}} \in \mathfrak{R}^{2 \mathrm{k}}$ be a vector of the $\mathrm{k}$ extracted image feature points, the task description as constraint is given by the following equation in the image space:

$$
\left(\ddot{\mathbf{s}}_{\mathrm{d}}-\ddot{\mathbf{s}}\right)+\mathbf{K}_{\mathrm{D}}\left(\dot{\mathbf{s}}_{\mathrm{d}}-\dot{\mathbf{s}}\right)+\mathbf{K}_{\mathrm{P}}\left(\mathbf{s}_{\mathrm{d}}-\mathbf{s}\right)=0
$$

where $\ddot{\mathbf{s}}_{\mathrm{d}}, \dot{\mathbf{s}}_{\mathrm{d}}$ and $\mathbf{s}_{\mathrm{d}}$ are the desired image space accelerations, velocities and positions, respectively. $\mathbf{K}_{\mathrm{P}}$ and $\mathbf{K}_{\mathrm{D}}$ are proportional and derivative gain matrices, respectively. This equation can be expressed in regard to image error in the following way:

$$
\ddot{\mathbf{s}}_{\mathrm{d}}+\mathbf{K}_{\mathrm{D}} \dot{\mathbf{e}}_{\mathrm{s}}+\mathbf{K}_{\mathrm{P}} \mathbf{e}_{\mathrm{s}}=\ddot{\mathbf{s}}_{\mathrm{r}}
$$

where $\mathbf{e}_{\mathrm{s}}$ and $\dot{\mathbf{e}}_{\mathrm{s}}$ are the image error and the time derivative of the error respectively. As stated, the variable $\ddot{\mathbf{s}}_{\mathrm{r}}$ denotes the reference image accelerations of our image space based controller. Replacing this variable into the dynamic visual servo controller, Equation (12), the control law is set by the following relationship:

$$
\boldsymbol{\tau}=\mathbf{W}^{-1 / 2}\left(\mathbf{L}_{\mathrm{J}} \mathbf{M}^{-1} \mathbf{W}^{-1 / 2}\right)^{+} \cdot\left(\ddot{\mathbf{s}}_{\mathrm{d}}+\mathbf{K}_{\mathrm{D}} \dot{\mathbf{e}}_{\mathrm{s}}+\mathbf{K}_{\mathrm{P}} \mathbf{e}_{\mathrm{s}}-\dot{\mathbf{L}}_{\mathrm{J}} \dot{\mathbf{q}}-\mathbf{L}_{\mathrm{J}} \mathbf{M}^{-1} \mathbf{F}_{\mathrm{cg}}\right)
$$

In Fig. 1 a scheme with the main components of the proposed framework is shown. In order to demonstrate an asymptotic tracking of the control law (15), some operations must be done. Firstly, the closed loop behavior is computed using Equation (3) as:

$$
\begin{aligned}
& \mathbf{M} \ddot{\mathbf{q}}-\mathbf{F}_{\mathrm{cg}}=\mathbf{W}^{-1 / 2}\left(\mathbf{L}_{\mathrm{J}} \mathbf{M}^{-1} \mathbf{W}^{-1 / 2}\right)^{+} . \\
& \left(\ddot{\mathbf{s}}_{\mathrm{d}}+\mathbf{K}_{\mathrm{D}} \dot{\mathbf{e}}_{\mathrm{s}}+\mathbf{K}_{\mathrm{P}} \mathbf{e}_{\mathrm{s}}-\dot{\mathbf{L}}_{\mathrm{J}} \dot{\mathbf{q}}-\mathbf{L}_{\mathrm{J}} \mathbf{M}^{-1} \mathbf{F}_{\mathrm{cg}}\right)
\end{aligned}
$$

Equation (16) can be simplified by pre-multiplying its left and right side by $\left(\mathbf{L}_{J} \mathbf{M}^{-1} \mathbf{W}^{-1 / 2}\right) \cdot \mathbf{W}^{1 / 2}$ :

$$
\mathbf{L}_{\mathrm{J}} \ddot{\mathbf{q}}=\ddot{\mathbf{s}}_{\mathrm{d}}+\mathbf{K}_{\mathrm{D}} \dot{\mathbf{e}}_{\mathrm{s}}+\mathbf{K}_{\mathrm{P}} \mathbf{e}_{\mathrm{s}}-\dot{\mathbf{L}}_{\mathrm{J}} \dot{\mathbf{q}}
$$

Using the relationship expressed in (6), it can be concluded that:

$$
\ddot{\mathbf{e}}_{\mathrm{s}}=-\mathbf{K}_{\mathrm{D}} \dot{\mathbf{e}}_{\mathrm{s}}-\mathbf{K}_{\mathrm{P}} \mathbf{e}_{\mathrm{s}}
$$

Therefore, when $\mathbf{L}_{\mathrm{J}}$ is full rank an asymptotic tracking is achieved of the visual servo controller expressed by Equation (15) in the tracking of an image trajectory.

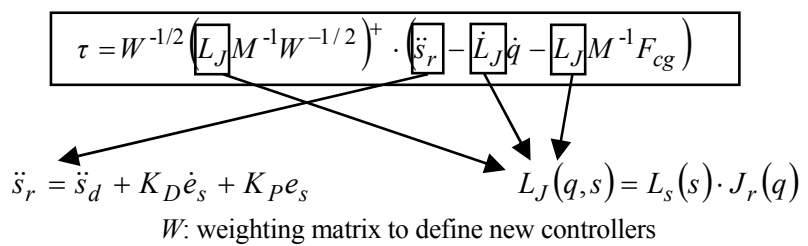

Fig. 1. Scheme of the main components of the proposed framework.

\subsection{Null-space resolution}

In this paper, a redundant joint structure is employed to track the image trajectory and hence $n>\eta$, being $\eta$ the dimension of where the path tracking is performed. In this case, the visual controller must guarantee the correct motion of end-effector and joints, avoiding singularities and/or possible chaotic behavior. Therefore, it is needed a joint function which stabilizes the robot without affecting the task performed in the image space. One possibility to do that is by defining a desired behavior that is only applied in the null space of the visual controller indicated in Equation (12). As it is shown in this last 
equation, the task space is defined by $\mathbf{W}^{-1 / 2}\left(\mathbf{L}_{\mathbf{J}} \mathbf{M}^{-1} \mathbf{W}^{-1 / 2}\right)^{+}$. Therefore, the term expressed as $\left[\mathbf{I}-\mathbf{W}^{-1 / 2}\left(\mathbf{L}_{J} \mathbf{M}^{-1} \mathbf{W}^{-1 / 2}\right)^{+}\left(\mathbf{L}_{\mathbf{J}} \mathbf{M}^{-1} \mathbf{W}^{-1 / 2}\right) \mathbf{W}^{1 / 2}\right] \cdot \boldsymbol{\tau}_{1}$ can be used to project the motor command $\boldsymbol{\tau}_{1}$ onto the null space of the dynamic visual servo task. Including this control in the proposed framework, the following control law can be obtained:

$$
\begin{aligned}
\boldsymbol{\tau}= & \mathbf{W}^{-1 / 2}\left(\mathbf{L}_{\mathrm{J}} \mathbf{M}^{-1} \mathbf{W}^{-1 / 2}\right)^{+} \cdot\left(\ddot{\mathbf{s}}_{\mathrm{r}}-\dot{\mathbf{L}}_{\mathrm{J}} \dot{\mathbf{q}}-\mathbf{L}_{\mathrm{J}} \mathbf{M}^{-1} \mathbf{F}_{\mathrm{cg}}\right)+ \\
& \mathbf{W}^{-1 / 2} \cdot\left[\mathbf{I}-\left(\mathbf{L}_{\mathbf{J}} \mathbf{M}^{-1} \mathbf{W}^{-1 / 2}\right)^{+}\left(\mathbf{L}_{\mathrm{J}} \mathbf{M}^{-1} \mathbf{W}^{-1 / 2}\right)\right] \mathbf{W}^{1 / 2} \cdot \boldsymbol{\tau}_{1}
\end{aligned}
$$

Thus, the task accomplishment is independent of the joint-space stabilization done by $\boldsymbol{\tau}_{1}$. Setting $\boldsymbol{\tau}_{1}=\mathbf{C}+\mathbf{G}+\boldsymbol{\tau}_{0}$, the Coriolis, centrifugal and gravitational forces can be compensated. Using this modification, the control law yields as follows:

$$
\begin{aligned}
\boldsymbol{\tau}= & \mathbf{W}^{-1 / 2}\left(\mathbf{L}_{\mathrm{J}} \mathbf{M}^{-1} \mathbf{W}^{-1 / 2}\right)^{+} \cdot\left(\ddot{\mathbf{s}}_{\mathrm{r}}-\dot{\mathbf{L}}_{\mathrm{J}} \dot{\mathbf{q}}\right)+\mathbf{C}+\mathbf{G} \\
& \mathbf{W}^{-1 / 2} \cdot\left[\mathbf{I}-\left(\mathbf{L}_{\mathrm{J}} \mathbf{M}^{-1} \mathbf{W}^{-1 / 2}\right)^{+}\left(\mathbf{L}_{\mathrm{J}} \mathbf{M}^{-1} \mathbf{W}^{-1 / 2}\right)\right] \mathbf{W}^{1 / 2} \cdot \boldsymbol{\tau}_{0}
\end{aligned}
$$

Next section will show the definition of the control signals $\tau_{0}$ and the one employed for chaos control.

\section{Modified control law using manipulability and a chaos compensation}

A singularity can be obtained in the manipulator Jacobian (see Equation (1)) when the joint structure is on a configuration in which certain end-effector velocities are not possible. Thus, the signal control $\tau_{0}$ will be employed to avoid possible singularities as a nullspace joint function which stabilizes the mechanical system without affecting the main task (tracking in the image space). This function employs the manipulability concept ${ }^{15}$ which measures the distance between the current configuration of the joint structure and the closest singular pose. A high manipulability is required to assure the ability of the system to move in arbitrary directions. The manipulability is defined as the square root of the determinant of the product of the manipulator Jacobian by its transpose, i.e.:

$$
\boldsymbol{\omega}_{\mathrm{r}}=\sqrt{\operatorname{det}\left(\mathbf{J}_{\mathrm{r}}^{\mathrm{T}} \mathbf{J}_{\mathrm{r}}\right)}
$$

The manipulability value is projected into the null-space of the controller by means its gradient and a damping term in joint space. This way, the control signal $\tau_{0}$ is established as follows:

$$
\boldsymbol{\tau}_{0}=\boldsymbol{\tau}_{\mathrm{Mp}}=-\mathrm{k}_{\mathrm{Dr}} \dot{\mathbf{q}}-\alpha_{\mathrm{r}} \nabla \boldsymbol{\omega}_{\mathrm{r}}
$$

where $\mathrm{k}_{\mathrm{Dr}}, \alpha_{\mathrm{r}}>0$ and $\nabla \omega_{\mathrm{r}}$ is the gradient of the manipulability ${ }^{16}$.

In regard to the chaos controller, a Delayed Feedback Control (DFC) method will be integrated in the framework ${ }^{17}$. This method generates a control signal proportional to the difference between the current state of the system, $\dot{\mathbf{q}}(\mathrm{t})$ and the state of the system delayed by one period, $\dot{\mathbf{q}}(\mathrm{t}-\varepsilon)$ :

$$
\boldsymbol{\tau}_{\mathrm{DFC}}=\mathbf{M} \lambda(\dot{\mathbf{q}}(\mathrm{t}-\varepsilon)-\dot{\mathbf{q}}(\mathrm{t}))
$$

where $\lambda$ is a constant gain to be determined, $\varepsilon$ is the feedback timedelay and $\mathbf{M}$ is the inertia matrix of the joint structure commented before. The adjustment of constants $\lambda$ and $\varepsilon$ is performed by using our previous algorithms ${ }^{12}$.
Adding these two terms to Equation (20), the general control law to dynamically visual servo control any joint structure with both redundant resolution and chaos compensation is obtained. This framework can be expressed as:

$$
\begin{aligned}
\boldsymbol{\tau}= & \boldsymbol{\tau}_{\mathrm{DFC}}+\mathbf{W}^{-1 / 2}\left(\mathbf{L}_{\mathrm{J}} \mathbf{M}^{-1} \mathbf{W}^{-1 / 2}\right)^{+} \cdot\left(\ddot{\mathbf{s}}_{\mathrm{r}}-\dot{\mathbf{L}}_{\mathrm{J}} \dot{\mathbf{q}}\right)+\mathbf{C}+\mathbf{G} \\
& \mathbf{W}^{-1 / 2} \cdot\left[\mathbf{I}-\left(\mathbf{L}_{\mathrm{J}} \mathbf{M}^{-1} \mathbf{W}^{-1 / 2}\right)^{+}\left(\mathbf{L}_{\mathrm{J}} \mathbf{M}^{-1} \mathbf{W}^{-1 / 2}\right)\right] \mathbf{W}^{1 / 2} \cdot \boldsymbol{\tau}_{\mathrm{Mp}}
\end{aligned}
$$

It should be pointed out that the control signals, $\boldsymbol{\tau}_{\mathrm{DFC}}$ and $\boldsymbol{\tau}_{\mathrm{Mp}}$, are not part of the contributions of this paper, but they can be incorporated easily to the presented framework.

\section{Precision study of the visual servo controllers}

\subsection{Dynamic visual servo controllers derived from the control framework}

Up to now, the control framework has been written depending on the weighting matrix $\mathbf{W}$. As stated, the choice of $\mathbf{W}$ plays an important role in the controller because determines how the torques are distributed over the joints. Moreover, different values of this matrix can simplify the product $\left(\mathbf{L}_{J} \mathbf{M}^{-1} \mathbf{W}^{-1 / 2}\right)^{+}$and consequently, the control law. Following, the new visual servoing control laws obtained from the choice of different values of $\mathbf{W}$ will be shown. A precision study of the derived controllers will be evaluated in the next subsection.

Considering $\mathbf{W}=\mathbf{M}^{-2}$ and replacing this value in the control framework expressed in Equation (24), the result yields:

$$
\begin{aligned}
\boldsymbol{\tau}= & \boldsymbol{\tau}_{\mathrm{DFC}}+\mathbf{M L}_{\mathrm{J}}^{+} \cdot\left(\ddot{\mathbf{s}}_{\mathrm{r}}-\dot{\mathbf{L}}_{\mathrm{J}} \dot{\mathbf{q}}\right)+\mathbf{C}+\mathbf{G}+ \\
& \mathbf{M} \cdot\left[\mathbf{I}-\mathbf{L}_{\mathbf{J}}^{+} \mathbf{L}_{\mathrm{J}}\right] \cdot \mathbf{M}^{-1} \cdot \boldsymbol{\tau}_{\mathrm{Mp}}
\end{aligned}
$$

This controller represents a direct visual servo control using inversion of the dynamic model of the joint structure.

Another value for the choice of $\mathbf{W}$ is $\mathbf{D} \mathbf{M}^{-2}$, where $\mathbf{D}$ is a diagonal positive matrix. This matrix allows distributing the torques on the joints, and therefore, large weights cause small torques. Using this value for $\mathbf{W}$, the controller from Equation (24) results as follows:

$$
\begin{aligned}
\boldsymbol{\tau}= & \boldsymbol{\tau}_{\mathrm{DFC}}+\left(\mathbf{D M}^{-2}\right)^{-1 / 2}\left(\mathbf{L}_{\mathrm{J}} \mathbf{M}^{-1}\left(\mathbf{D M}^{-2}\right)^{-1 / 2}\right)^{+}\left(\ddot{\boldsymbol{s}}_{\mathrm{r}}-\dot{\mathbf{L}}_{\mathrm{J}} \dot{\mathbf{q}}\right)+\mathbf{C}+\mathbf{G} \\
& +\left(\mathbf{D M}^{-2}\right)^{-1 / 2} \cdot\left[\mathbf{I}-\left(\mathbf{L}_{\mathbf{J}} \mathbf{M}^{-1}\left(\mathbf{D} \mathbf{M}^{-2}\right)^{-1 / 2}\right)^{+}\left(\mathbf{L}_{\mathbf{J}} \mathbf{M}^{-1}\left(\mathbf{D M}^{-2}\right)^{-1 / 2}\right)\right] . \\
& \left(\mathbf{D M}^{-2}\right)^{1 / 2} \cdot \boldsymbol{\tau}_{\mathrm{Mp}}
\end{aligned}
$$

Applying the pseudo inverse as $\mathbf{Q}^{+}=\mathbf{Q}^{\mathrm{T}}\left(\mathbf{Q}^{\cdot} \mathbf{Q}^{\mathrm{T}}\right)^{-1}$ and simplifying this equation, the control law yields as:

$$
\begin{aligned}
\boldsymbol{\tau}= & \boldsymbol{\tau}_{\mathrm{DFC}}+\mathbf{M D}^{-1} \mathbf{L}_{\mathrm{J}}{ }^{\mathrm{T}} \cdot\left(\mathbf{L}_{\mathrm{J}} \mathbf{D}^{-1} \mathbf{L}_{\mathrm{J}}{ }^{\mathrm{T}}\right)^{-1}\left(\ddot{\boldsymbol{s}}_{\mathrm{r}}-\dot{\mathbf{L}}_{\mathrm{J}} \dot{\mathbf{q}}\right)+\mathbf{C}+\mathbf{G}+ \\
& \mathbf{M D}^{-1 / 2} \cdot\left[\mathbf{I}-\mathbf{D}^{-1 / 2} \mathbf{L}_{\mathrm{J}}{ }^{\mathrm{T}}\left(\mathbf{L}_{\mathrm{J}} \mathbf{D}^{-1} \mathbf{L}_{\mathrm{J}}{ }^{\mathrm{T}}\right)^{-1} \mathbf{L}_{\mathrm{J}} \cdot \mathbf{D}^{-1 / 2}\right] \cdot \mathbf{D}^{1 / 2} \mathbf{M}^{-1} \cdot \boldsymbol{\tau}_{\mathrm{Mp}}
\end{aligned}
$$

Additionally, new controllers can be obtained using different values of $\mathbf{W}$. According to $\mathrm{Udwadia}^{14}$, an important value for $\mathbf{W}$, due to its physical interpretation, is $\mathbf{W}=\mathbf{M}^{-1}$, since it is consistent with the principle of d'Alembert. Furthermore, the use of $\mathbf{W}=\mathbf{I}$ simplifies the control law. In the next section the controllers obtained when $\mathbf{W}=$ $\mathbf{M}^{-1}, \mathbf{W}=\mathbf{D} \mathbf{M}^{-1}, \mathbf{W}=\mathbf{I}, \mathbf{W}=\mathbf{D}, \mathbf{W}=\mathbf{M}^{-2}$, and $\mathbf{W}=\mathbf{D} \mathbf{M}^{-2}$ are evaluated and compared with classical direct visual servoing like the 
proposed in previous works ${ }^{4}$.

\subsection{Experimental results}

In this section, a set of experiments is presented to illustrate the precision performance of different direct visual servoing control laws obtained using the proposed framework. In order to evaluate the precision and properties of the generated controllers the eye-in-hand joint structure presented in Fig. 2 is employed. A Gigabit Ethernet TM6740GEV camera is used, which acquires 200 images every second with a resolution of $640 \times 480$ pixels. The joint structure has four rotational joints ( $\left.\mathrm{q}_{0}, \mathrm{q}_{1}, \mathrm{q}_{2}, \mathrm{q}_{3}\right)$ whose actuators are four geared DC motors but only three joints $\left(\mathrm{q}_{1}, \mathrm{q}_{2}, \mathrm{q}_{3}\right)$ are employed for the experiments. The camera is supposed to be previously calibrated and the camera intrinsic parameters are $\left(\mathrm{u}_{0}, \mathrm{v}_{0}\right)=(298,225) \mathrm{px}$, and $\left(\mathrm{f}_{\mathrm{u}}\right.$, $\left.f_{v}\right)=(1082.3,1073.7) p x$ (position of the optical center $\left(u_{0}, v_{0}\right)$ and the focal length in the $\mathrm{x}$ and $\mathrm{y}$ directions, respectively). For the sake of clarity, the eye in hand camera is observing one visual feature performing a planar movement with respect to the robot at a distance of $1 \mathrm{~m}(\mathrm{k}=1)$. In all the experiments presented in this section the image space gain settings are $\mathbf{K}_{\mathrm{P}}=0.1 \mathbf{I}$ and $\mathbf{K}_{\mathrm{D}}=0.5 \mathbf{I}$ (the proportional and derivative matrices of the image path).

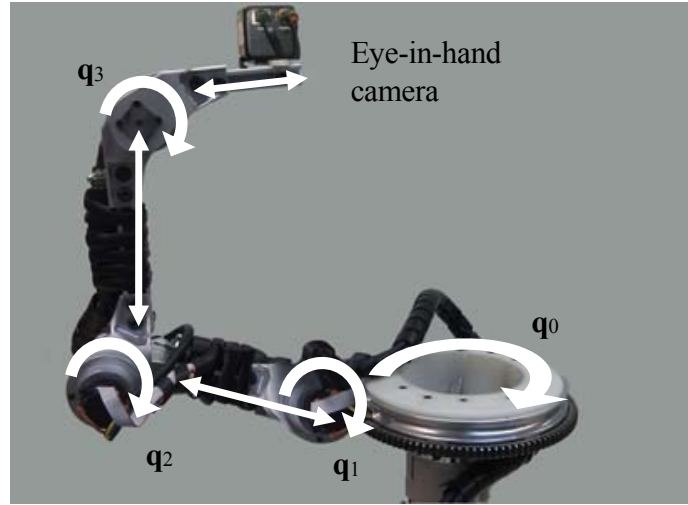

Fig. 2. Robot employed in the experimental results.

\subsubsection{Experiment 1}

In this section the obtained visual servoing control laws with chaos compensation are evaluated during the tracking of the periodic image trajectory defined by the following equations:

$$
\mathbf{s}_{\mathrm{d}}=\left[\begin{array}{l}
f_{\mathrm{xd}} \\
f_{\mathrm{yd}}
\end{array}\right]=\left[\begin{array}{l}
320+166 \cos (\omega \mathrm{t}+\pi / 4) \\
265+160 \sin (\omega \mathrm{t}+\pi / 4)
\end{array}\right]
$$

where the parameter $\omega$ determines the tracking velocity.

Firstly, in order to evaluate the tracking system with chaos compensation a medium velocity is considered $(\omega=1 \mathrm{rad} / \mathrm{s})$. Fig. 3 represents in blue the desired image trajectory obtained when $\mathbf{W}=\mathbf{M}^{-2}, \mathbf{W}=\mathbf{D M}^{-2}, \mathbf{W}=\mathbf{M}^{-1}, \mathbf{W}=\mathbf{D M}^{-1}, \mathbf{W}=\mathbf{I}$, and $\mathbf{W}=\mathbf{D}$. A correct behavior is obtained for all the controllers; however, it can be observed that the trajectory is more accurately tracked when $\mathbf{W}=\mathbf{M}^{-2}$ and $\mathbf{W}=\mathbf{M}^{-1}$. As previously indicated, when $\mathbf{W}=\mathbf{D M}^{-2}, \mathbf{W}=\mathbf{D}$ or $\mathbf{W}$ $=\mathbf{D M}^{-1}$ the value of $\mathbf{D}$ allows us to indicate which joints will support high loads. In this experiment, the weight value corresponding to the first joint is twice the weight corresponding to the second and third joints. Fig. 4 represents the joint configurations of the robot during the tracking and Fig. 5 represents the robot configurations considering $\mathbf{W}=\mathbf{M}^{-2}$ and without introducing the chaos controller. Comparing both figures, it can be observed that using the proposed controllers the chaos behavior is compensated and a periodic joint trajectory is obtained during the tracking of the periodic image trajectory.
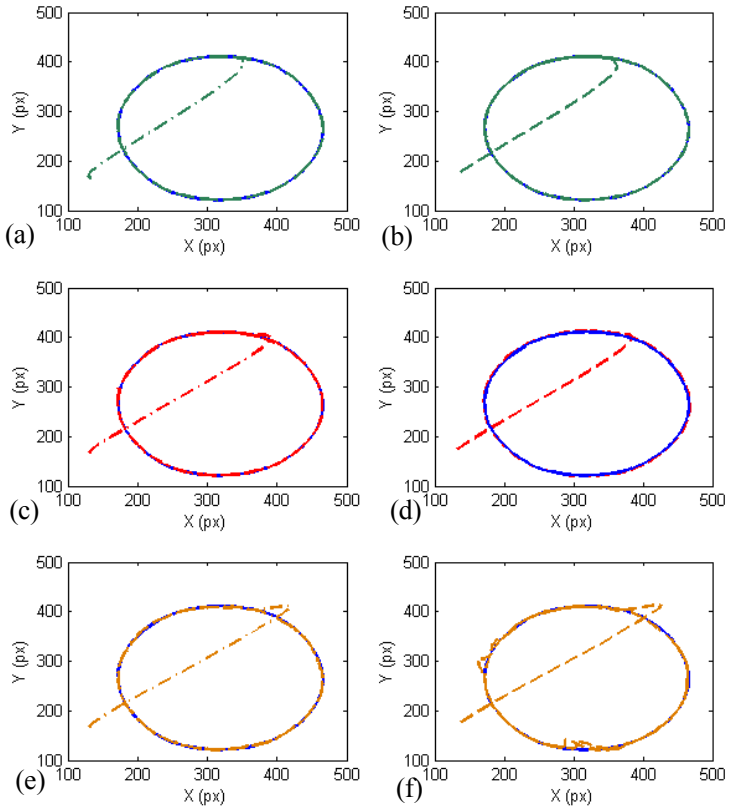

Fig. 3. Experiment 1. Desired image trajectory (blue) and obtained image trajectories considering: (a) $\mathbf{W}=\mathbf{M}^{-2}$. (b) $\mathbf{W}=\mathbf{D M}^{-2}$. (c) $\mathbf{W}=\mathbf{M}^{-1}$. (d) $\mathbf{W}=$ $\mathbf{D M}^{-1}$. (e) $\mathbf{W}=\mathbf{I}$. (f) $\mathbf{W}=\mathbf{D}$.
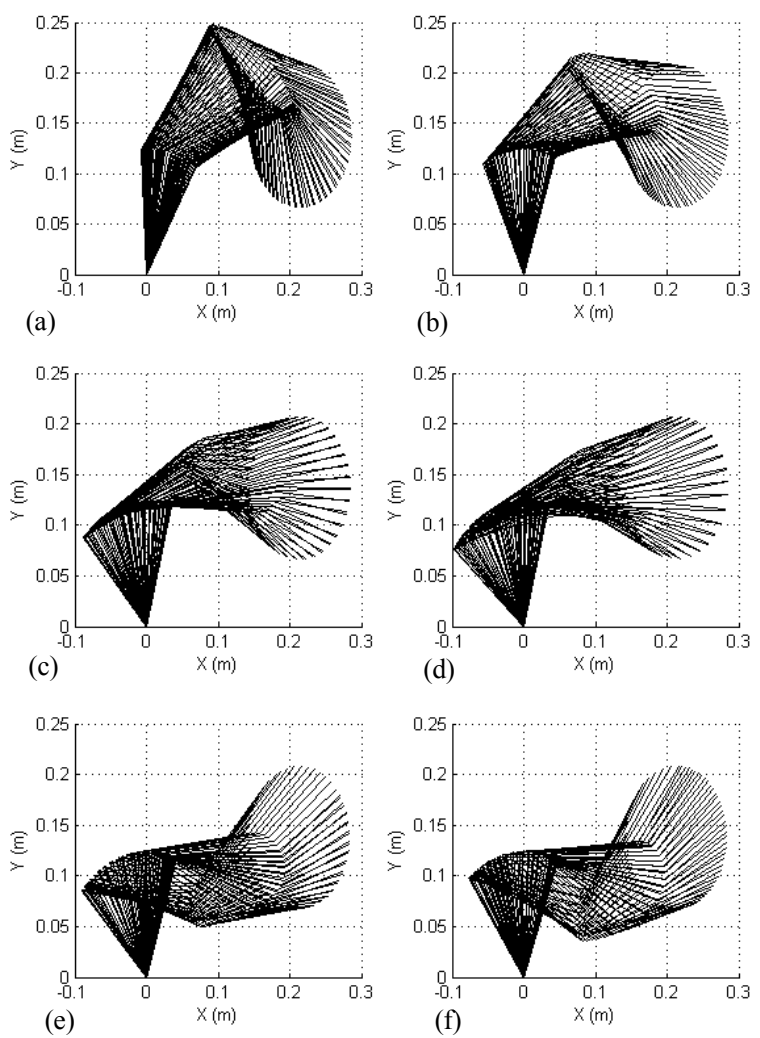

Fig. 4. Experiment 1 . Obtained $3 \mathrm{D}$ trajectory during the tracking considering (a) $\mathbf{W}=\mathbf{M}^{-2}$. (b) $\mathbf{W}=\mathbf{D} \mathbf{M}^{-2}$. (c) $\mathbf{W}=\mathbf{M}^{-1}$. (d) $\mathbf{W}=\mathbf{D} \mathbf{M}^{-1}$. (e) $\mathbf{W}=\mathbf{I}$. (f) $\mathbf{W}=\mathbf{D}$. 


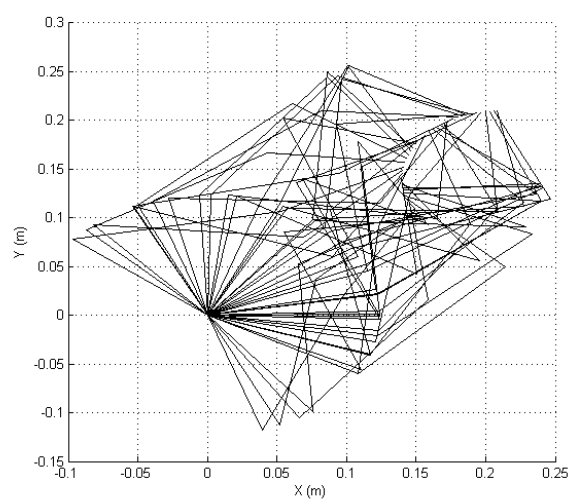

Fig. 5. Experiment 1. Robot configurations during the tracking without chaos $\operatorname{control}\left(\mathbf{W}=\mathbf{M}^{-2}\right)$.

In order to show more clearly the tracking precision, Table 1 represents the mean error in pixels during the tracking of the previous indicated trajectory considering different tracking velocities. The mean image error obtained by using previous classical direct visual controllers $^{4}$ is also included in this table. As it can be seen in Table 1, the image error is lower when $\mathbf{W}=\mathbf{M}^{-1}$ or $\mathbf{W}=\mathbf{M}^{-2}$ is employed. Furthermore, in these cases the performance is clearly improved with respect to the previous controllers ${ }^{4}$. Finally, if we consider $\omega=3 \mathrm{rad} / \mathrm{s}$ an image error about 7 pixels is obtained in all the cases.

Table 1 Mean image error (Pixels). Experiment 1.

\begin{tabular}{|c|c|c|c|}
\hline $\mathbf{W}$ & $\omega=0.5 \mathrm{rad} / \mathrm{s}$ & $\omega=1 \mathrm{rad} / \mathrm{s}$ & $\omega=2 \mathrm{rad} / \mathrm{s}$ \\
\hline $\mathbf{M}^{-2}$ & 2.14 & 2.12 & 3.84 \\
\hline $\mathbf{D M}^{-2}$ & 2.12 & 2.43 & 3.74 \\
\hline $\mathbf{M}^{-1}$ & 2.12 & 2.95 & 2.39 \\
\hline $\mathbf{D M}^{-1}$ & 2.42 & 3.10 & 3.34 \\
\hline $\mathbf{I}$ & 3.81 & 3.22 & 4.40 \\
\hline $\mathbf{D}$ & 3.45 & 3.66 & 4.81 \\
\hline $\begin{array}{c}\text { Previous } \\
\text { direct visual } \\
\text { controller }\end{array}$ & 2.25 & 3.20 & 4.12 \\
\hline
\end{tabular}

\subsubsection{Experiment 2}

In order to evaluate the tracking considering a more complex trajectory, all the controllers are tested during the tracking of a trajectory with abrupt changes (see Fig. 6). In the experiments represented in this last figure, a medium tracking velocity is considered $(\omega=1 \mathrm{rad} / \mathrm{s})$. As in the previous section, a correct tracking is achieved in the image space for all the six controllers and the best behaviour is obtained when $\mathbf{W}=\mathbf{M}^{-2}$ or $\mathbf{W}=\mathbf{M}^{-1}$. As it was indicated in the previous section, the matrix $\mathbf{D}$ is a diagonal matrix and the weight value corresponding to the first joint is twice the weight corresponding to the second and third joints. In order to demonstrate the effect obtained by using this matrix, in Fig. 7 the torques which were obtained when $\mathbf{W}=\mathbf{M}^{-2}$ and $\mathbf{W}=\mathbf{D M}^{-2}$ are represented. Comparing Fig. 7.a and Fig. 7.b, it can be observed that when $\mathbf{W}=$ $\mathbf{D M}^{-2}$ lower torques in the first joint are obtained. Therefore, this diagonal matrix can be employed to distribute the torques and to diminish the effort in the desired joints.
Fig. 8 represents the robot joint configurations achieved during the tracking in the $3 \mathrm{D}$ space. As it can be observed, the chaos is compensated (see Fig. 9 which represents the robot joints configurations when chaos control is not applied).

Finally, in order to indicate more clearly the precision and errors during the task, Table 2 indicates the mean error in pixels during the tracking considering different tracking velocities. From this table, similar conclusions can be extracted. The highest precision is obtained when $\mathbf{W}=\mathbf{M}^{-1}$ or $\mathbf{W}=\mathbf{M}^{-2}$. In contrast, when $\mathbf{W}=\mathbf{I}$ the controller is simpler and easier to implement. Additionally, the use of $\mathbf{W}=\mathbf{D M}^{-2}, \mathbf{W}=\mathbf{D}$ or $\mathbf{W}=\mathbf{D M}^{-1}$ also introduces small errors during the tracking but it allows us to indicate which joints will support high loads. Thus, the proposed framework can be employed to generate the controllers depending on the tracking requirements. Furthermore, the framework can be extended in order to generate new direct visual servo controllers with other properties depending the value of $\mathbf{W}$.
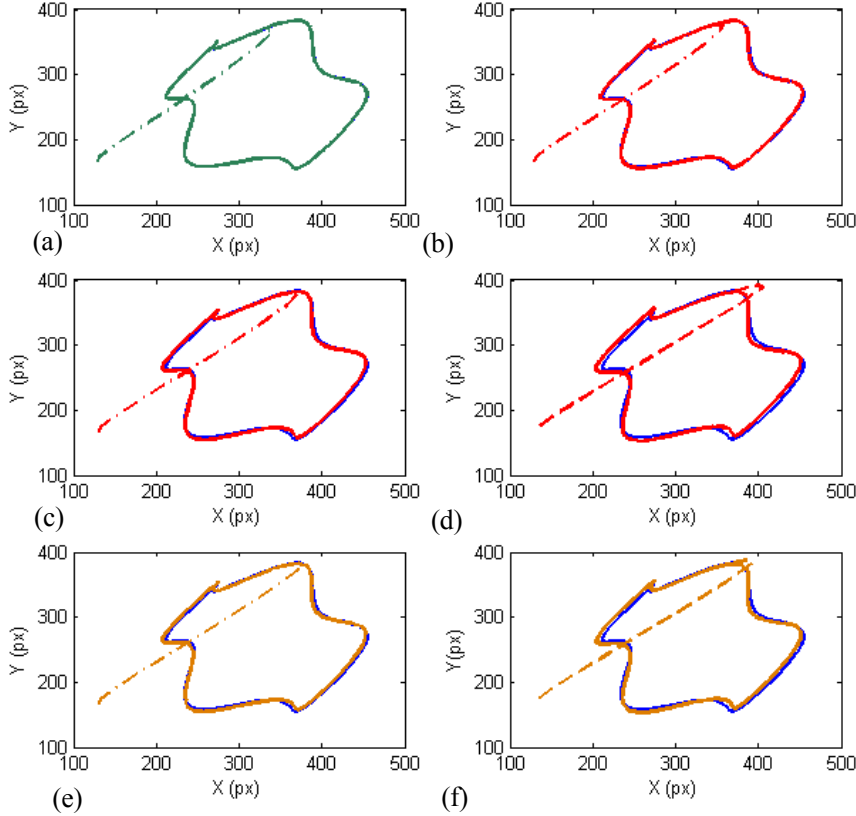

(d)

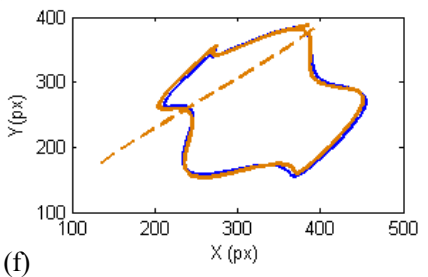

Fig. 6. Experiment 2. Desired image trajectory (blue) and obtained image trajectories considering: (a) $\mathbf{W}=\mathbf{M}^{-2}$. (b) $\mathbf{W}=\mathbf{D M}^{-2}$. (c) $\mathbf{W}=\mathbf{M}^{-1}$. (d) $\mathbf{W}=$ $\mathbf{D M}^{-1}$. (e) $\mathbf{W}=\mathbf{I}$. (f) $\mathbf{W}=\mathbf{D}$.
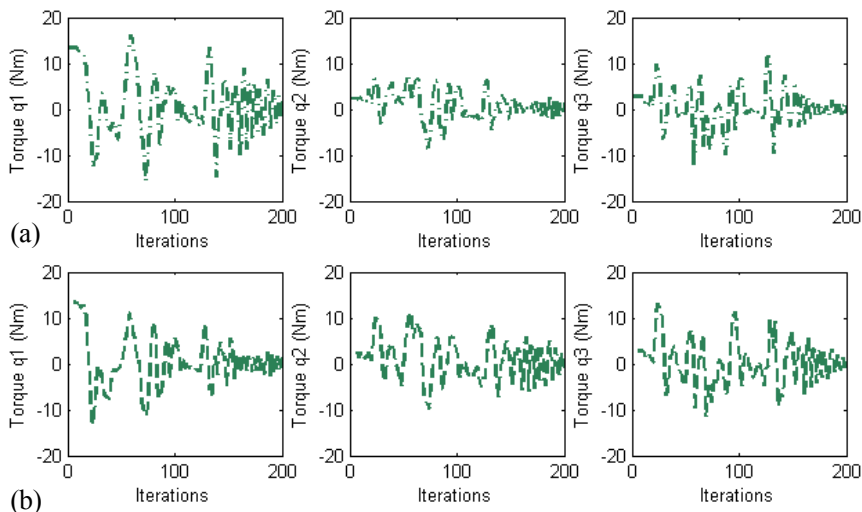

Fig. 7. Experiment 2. Torques obtained during the experiment when: (a) $\mathbf{W}=$ $\mathbf{M}^{-2}$. (b) $\mathbf{W}=\mathbf{D M}^{-2}$. 

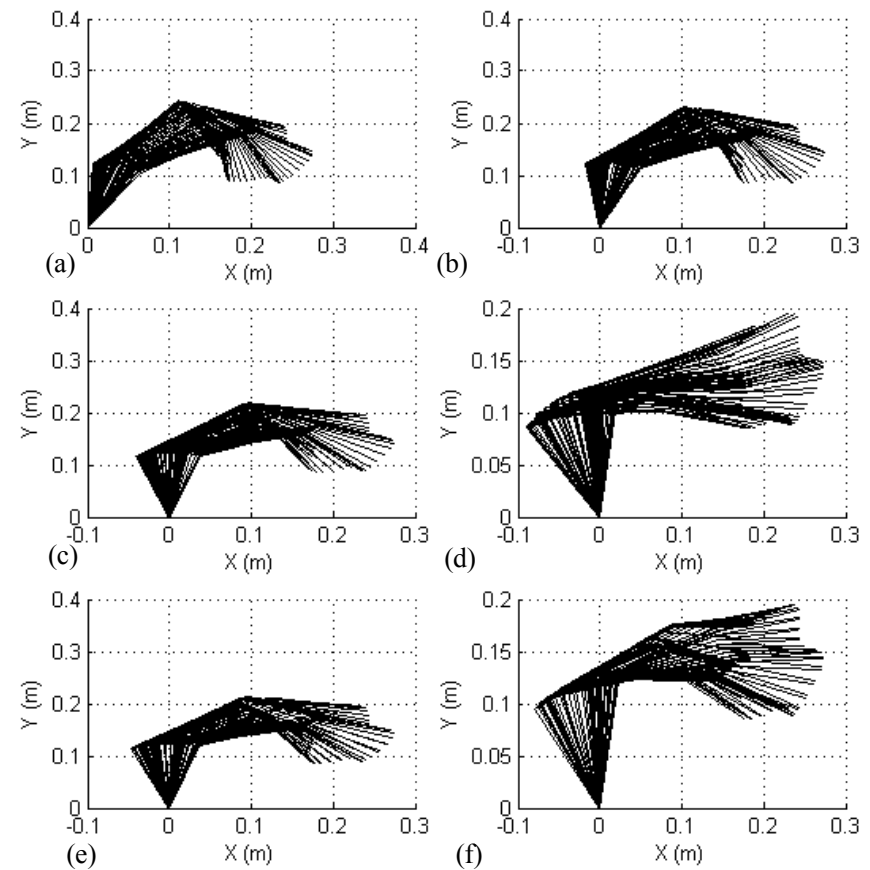

Fig. 8. Experiment 2. Obtained 3D trajectory during the tracking considering (a) $\mathbf{W}=\mathbf{M}^{-2}$. (b) $\mathbf{W}=\mathbf{D} \mathbf{M}^{-2}$. (c) $\mathbf{W}=\mathbf{M}^{-1}$. (d) $\mathbf{W}=\mathbf{D} \mathbf{M}^{-1}$. (e) $\mathbf{W}=\mathbf{I}$. (f) $\mathbf{W}=\mathbf{D}$.

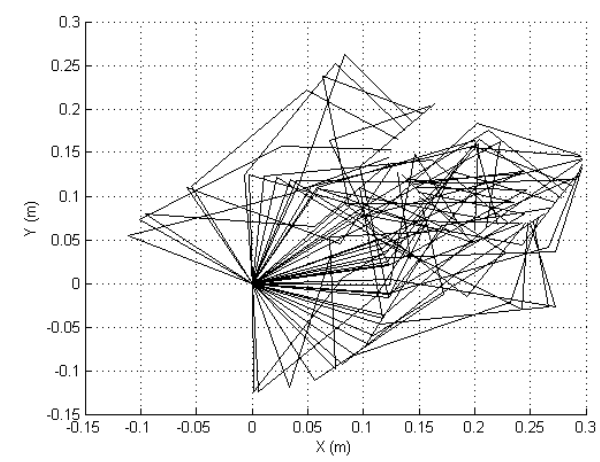

Fig. 9. Experiment 2. Robot configurations during the tracking without chaos control $\left(\mathbf{W}=\mathbf{M}^{-1}\right)$.

Table 2 Mean image error (Pixels). Experiment 2.

\begin{tabular}{|c|c|c|c|}
\hline $\mathbf{W}$ & $\omega=0.5 \mathrm{rad} / \mathrm{s}$ & $\omega=1 \mathrm{rad} / \mathrm{s}$ & $\omega=2 \mathrm{rad} / \mathrm{s}$ \\
\hline $\mathbf{M}^{-2}$ & 1.54 & 1.95 & 3.00 \\
\hline $\mathbf{D M}^{-2}$ & 2.41 & 2.63 & 3.55 \\
\hline $\mathbf{M}^{-1}$ & 1.34 & 1.92 & 2.55 \\
\hline $\mathbf{D M}^{-1}$ & 3.87 & 4.00 & 4.48 \\
\hline $\mathbf{I}$ & 1.75 & 2.22 & 3.12 \\
\hline $\mathbf{D}$ & 4.45 & 4.88 & 5.21 \\
\hline $\begin{array}{c}\text { Previous } \\
\text { direct visual } \\
\text { controller }\end{array}$ & 1.5 & 2.0 & 3.1 \\
\hline
\end{tabular}

\section{Conclusions}

In this paper, authors have presented the use of an optimal control approach which allows defining new dynamic visual controllers in order to carry out the guidance of any serial link structure with both redundant resolution and chaos compensation. Making use of both the dynamic robot model and the definition of the image trajectory as task description, different image-based dynamic visual servoing systems are defined. The proposed approach allows derivation of previously known dynamic visual controllers, and permits the development of new ones for any mechanical system with redundancy.

The implementation of some derivate controllers on a real robotic system has been carried out. As stated, new controllers are generated by using the proposed framework. Each controller has different precision and behavior in both the task and joint space. Therefore, the proposed framework can be employed in order to generate new direct visual servoing controllers with the performance required for a given application.

\section{ACKNOWLEDGEMENT}

This work was funded by the Spanish Ministry of Economy, the European FEDER funds and the Valencia Regional Government, through the research projects DPI2012-32390 and PROMETEO/2013/085.

\section{REFERENCES}

1. Andolfatto L., Lavernhe S. and Mayer JRR., "Evaluation of servo, geometric and dynamic error sources on five-axis high-speed machine tool," International Journal of Machine Tools \& Manufacture, Vol. 51, pp. 787-96, 2011.

2. Song, W., Wang, G., Xiao, J., Wang, G. and Hong, Y., "Research on multi-robot open architecture of an intelligent $\mathrm{CNC}$ system based on parameter-driven technology," Robotics and ComputerIntegrated Manufacturing, Vol. 28, pp. 326-333, 2012.

3. Chaumette, F. and Hutchinson, S., "Visual Servo Control, Part I: Basic Approaches," IEEE Robotics and Automation Magazine, Vol. 13, No. 4, pp. 82-90, 2006.

4. Pomares, J., Corrales, J. A., Garcia, G. J. and Torres, F., "Direct Visual Servoing to Track Trajectories in Human-Robot Cooperation," International Journal of Advanced Robotic Systems, Vol. 8, No. 4, pp. 129-138, 2011.

5. Khatib, O., "Dynamic Control of Manipulators in Operational Space," Proceedings of the 6th IFTOMM Congress on Theory of Machines and Mechanisms, pp. 1-10, 1983.

6. Xian, B., Queiroz, M., Dawson, D. and Walker, I., "Task Space Tracking Control of Redundant Robot Manipulators via Quaternion Feedback," Proceedings of IEEE International Conference on Control Applications, pp. 363-368, 2001.

7. Zergeroglu, E., Dawson, D. M., Walker, I. and Behl, A., "Nonlinear Tracking Control of Kinematically Redundant Robot Manipulators," Proceedings of the American Control Conference, pp. 2513-2517, 2000.

8. Tatlicioglu, E., Braganza, D., Burg, T. C. and Dawson, D. M., "Adaptive Control of Redundant Robot Manipulators with SubTask Objectives," Robotica, Vol. 27, pp. 873-881, 2009. 
9. Lin, C. J., "Motion Planning of Redundant Robots by Perturbation Method," Mechatronics, Vol. 14, pp. 281-297, 2004.

10. Nakanishi, J., Cory, R., Mistry, M., Peters, J. and Schaal, S., "Operational Space Control: A Theoretical and Empirical Comparison,". International Journal of Robotics Research, Vol. 27, No. 6, pp. 737-757, 2008.

11. Kumar, N., Borm, J., Panwar, V. and Chai, J., "Tracking control of redundant robot manipulators using RBF neural network and an adaptive bound on disturbances," International Journal of Precision Engineering and Manufacturing, Vol. 13, No. 8, pp 1377-1386, 2012.

12. Pomares, J., Perea, I. and Torres, F., "Dynamic Visual Servoing With Chaos Control for Redundant Robots," IEEE/ASME Transactions on Mechatronics, Vol. 19, No. 2, pp 423-431, 2014.

13. Sciavicco, L., Siciliano, B. and Sciavicco, B., "Modelling and control of robot manipulators," Springer-Verlag London, United Kingdom, 2002.

14. Udwadia, F. E., "A new perspective on tracking control of nonlinear structural and mechanical systems," Proceedings of the Royal Society of London Series A, pp. 1783-1800, 2003.

15. Yoshikawa, T., "Manipulability of Robotic Mechanisms," International Journal of Robotics Research, Vol. 4, No. 2, pp. 3-9, 1985.

16. Park, J., Chung, W. and Youm, Y., "Computation of Gradient of Manipulability for Kinematically Redundant Manipulators Including Dual Manipulators Systems," Transaction on Control Automation and Systems Engineering, Vol. 1, No. 1, pp. 8-15, 1999.

17. Pyragas, K., "Delayed feedback control of chaos," Philosophical Transactions of the Royal Society, Vol. 364, No. 1846, pp. 23092334, 2006. 